\title{
Are the modern computer simulations a substitute for physical models? The Antikythera case
}

\author{
Manos Roumeliotis ${ }^{* \dagger}$ \\ University of Macedonia \\ E-mail: manosduom.ar
}

\begin{abstract}
Computer simulation is a modeling and verification tool that is used primarily for mathematical, dynamic, arithmetic models. It is particularly suitable for the study of complex multivariable systems, the verification of a design before the machine is actually built, and the analysis of probabilistic systems. In the case of the Antikythera Mechanism we are faced with a completely deterministic system with a single input. However, the operation of this fascinating mechanism is not known in its entirety, derived only by the parts of the mechanism that have been found and studied. Since it is considered by many to be the first portable computer ever built, it is very appropriate to use a modern date computer simulation for validating the functional description of the machine, as well as estimating the possible operation of missing parts. From identifying errors in Derek de Solla Price's drawings, to understanding the implementation of the Hipparchos lunar orbit observations, to calculating the exact tolerances required for the construction of a physical model, the simulation of the mechanism has been proven invaluable. In addition, computer simulation is readily available to any researcher or scholar, and can even be used for educational purposes, whenever a physical model is unavailable. Even if a physical model is available and accessible, it can only be used for demonstration purposes. On the contrary, a simulation model can be easily calibrated to exact dates by computationally "turning" the gears to the required time. Then, it can be used for calculating astronomical phenomena in accordance to the mechanism's original design, without the need for cumbersome adjustments. Finally, as new data about the Antikythera Mechanism become available, the simulation model can be easily reconfigured to take into account the new findings, while a physical model is much more difficult to modify.
\end{abstract}

From Antikythera to the Square Kilometre Array: Lessons from the Ancients

12-15 June 2012

Kerastari, Greece

\footnotetext{
* Speaker.

$\dagger$ Prof. Manos Roumeliotis is a Professor of Computer Architecture at the Department of Technology Management of the University of Macedonia, Thessaloniki, Greece.
} 

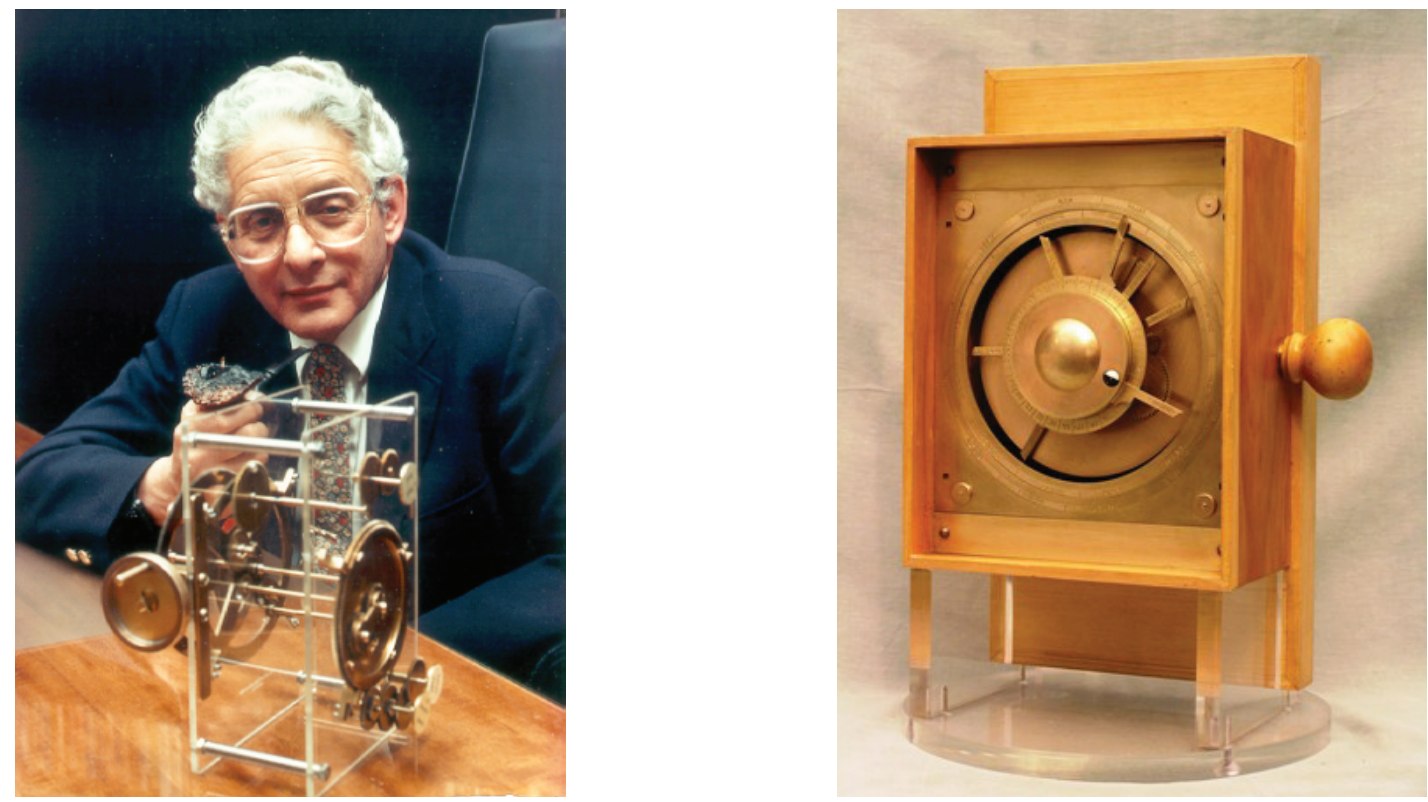

Figure 1: Physical Models of the mechanism, Price's (left) and Wright's (right).

\section{Introduction}

Derek de Solla Price studied the Antikythera mechanism for 25 years during which he published his now famous papers of 1959 [团] and 1974 [[]]. Although quite a few researchers before Price presented various hypotheses about the mechanism, it was Price who was able to meticulously count the teeth of each gear based on the radiographs of Dr. Karakalos, and give the first detailed description of the mechanism's operation. Today, based on the newer finding of the Antikythera Research Project [ [ $]$, we know that some of his conjecturers, like the differential turntable, are not correct. Nevertheless, Price built the solid foundation on which any further research on the mechanism is based.

Apart from the mechanical drawings he drafted, and in order to have a better conceptual idea of how the mechanism worked, Price had a physical model of the Antikythera mechanism built. Price with his model is shown in Figure 1 on the left. Neither his model, nor most of the physical models that were created subsequently operate. This is mainly attributed to the fact that most of the gears' bearings cannot be clearly distinguished in the radiographs or tomographies and, to be able to clearly display the inner gears, most reconstructions make the mounting plates less robust than they should be [四]. The only, until now, physical reconstruction that operates flawlessly is the one made by Michael Wright [W] but it slightly deviates from the original design by adding some mounting plates. Wright's model is shown in Figure $\mathbb{W}$ on the right. Other more recent operational physical models demonstrate only the functions of the mechanism, without being accurate reconstructions.

Although physical models, albeit mostly non-operational, were built early in the study of the mechanism, simulation models had to wait for the advancement in both computer hardware and software tools before they emerged. The first computer simulation was developed by Robert Morris who used a block diagram compiler to create an animated version of the mechanism's gears for a vector graphics system [ [ $]$. This was an experimental model and not a real simulation of the 


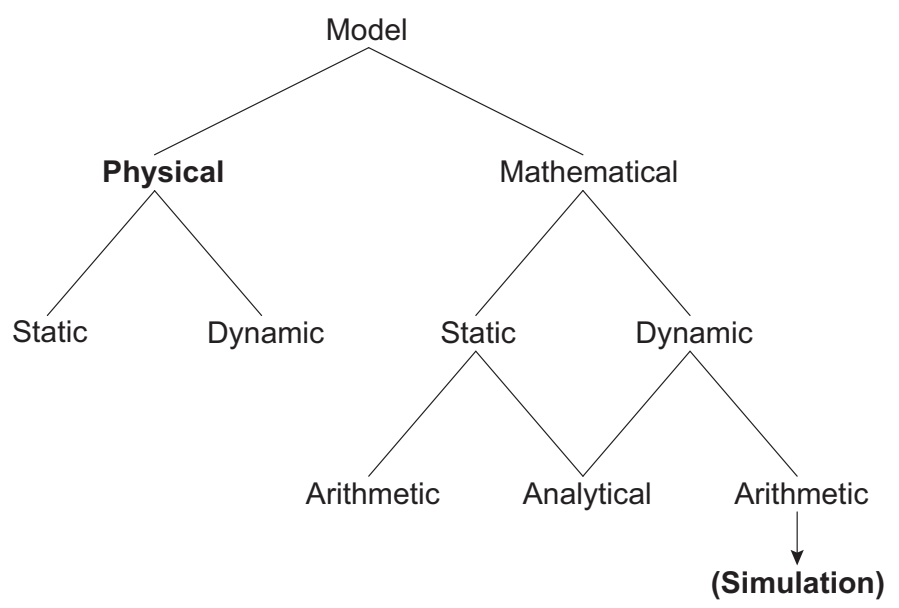

Figure 2: Classification of models.

mechanism, since at the time of its development (around 1980), graphics terminals were not readily available.

This paper examines accurate simulations and animations of the Antikythera mechanism, and compares the simulations to the physical models of the device. The study takes into account purpose, scope, availability, and accessibility of the models, to demonstrate that simulations do not simply provide an alternative to the physical models, but an invaluable tool for research and dissemination of the research's results.

\subsection{Modeling and Simulation}

The taxonomy of models is given in Figure $\square[\square]$. Models are classified into physical or mathematical. Both of these types of models are subdivided into static and dynamic depending on wether they change in time or not. Then, mathematical models are further subdivided into analytical and arithmetic models depending on whether there exist analytical equations to describe them or not. As shown in this figure, simulation is mainly used for arithmetic, dynamic, mathematical models.

According to the above classification, it would seem more appropriate to built a physical model for a deterministic mechanical system like the Antikythera Mechanism, rather than a computer simulation. What cannot be derived from this taxonomy is the fact that computer simulation models of certain systems have a great educational value, being able to demonstrate the complex operation of a system through direct visualization. This is the reason we frequently see animation and simulation models of engines, machines, and devices.

However, as will be shown in the following paragraphs, apart from its obvious educational value, computer simulation of the Antikythera Mechanism has contributed to the validation of the mathematical models, the identification of errors in the physical designs, and the verification of operational descriptions.

\section{Model Verification}

The study of the Antikythera mechanism by de Solla Price resulted in a functional description 


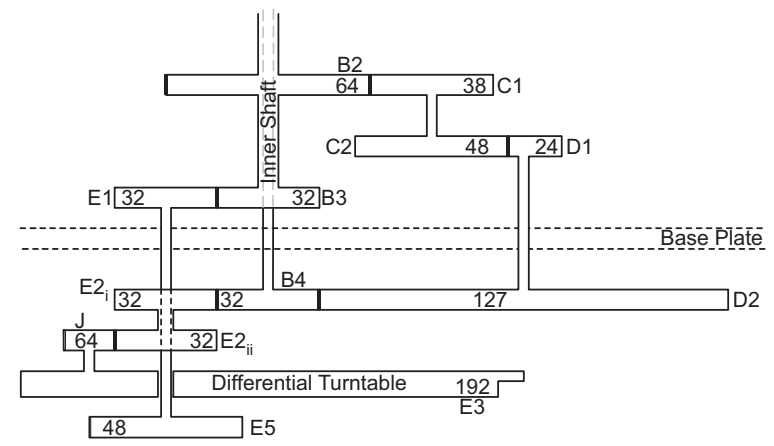

(a)

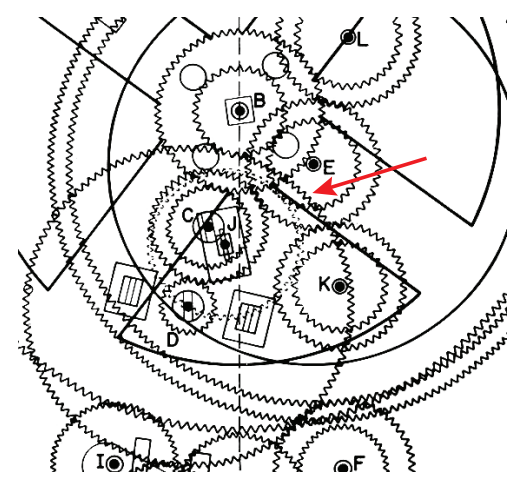

(b)

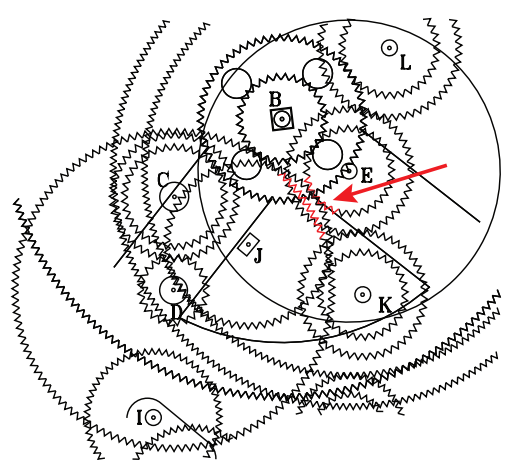

(c)

Figure 3: Recalculation of Price's gear positions. (a) Part of the sectional diagram. (b) Part of Price's planar view, (c) Repositioning of the gears.

which included detailed drawings of the mechanism. To verify these drawings and visualize the operation of the mechanism we tried to create a computer model of the mechanism. The transformation of the drawings into computer constructs revealed a error in the drawings as demonstrated in Figure [3.

The drawing on the top (Figure B(a)) is part of the sectional diagram as given by Morris in [3] . As can be seen, gear $\mathrm{E} 2{ }_{i}$ engages gear B4 which engages gear D2. It follows that gears E2 ${ }_{i}$. B4, and D2 are on the same plane. However, in the planar diagram shown in Figure B(b), gears E ${ }_{i}$ and D2 overlap at the position pointed by the red arrow. As this cannot possibly be the case, the design was corrected for the computer simulation model, by slightly modifying the position of the gears' axes. In the final model, the gears have quite some clearance as shown in Figure B)(c) by the red arrow and the red gear teeth.

In addition to static representation of the model's dimensions, computer simulation models of the Antikythera Mechanism require the accurate specification of either rotation equations for the various parts (mainly gears), or the generation of accurate positional data for each subpart. As these specifications are based on the physical measurements of the mechanism, they can be used to verify the obtained data. As an example, consider the simulation modeling of the Hipparchos gear train [2]. The rotation of gear k1 (the one with the pin) can be modeled simply be the relative rotation of the gears driving $\mathrm{k} 1$. However, the rotation of gear $\mathrm{k} 2$ is rather complicated. The best 


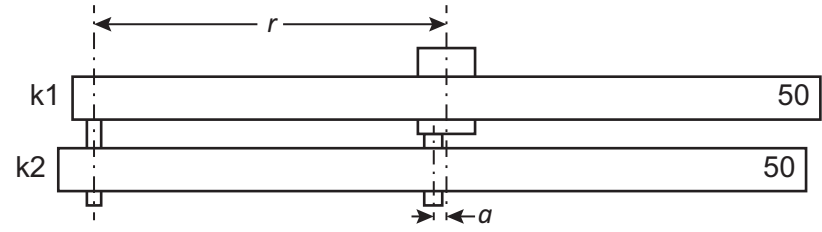

(a)

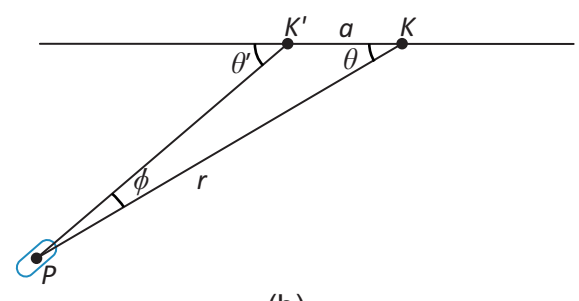

(b)

Figure 4: Calculation of the relative angle of the slotted Hipparchos gear.

way to model it for the simulation, is to calculate the rotational angle of gear k2 relative to the rotational angle of gear k1. Figure $\mathrm{H}$ shows the sectional diagram of gears k1 and k2 (Figure G(a)) and the calculation of angle $\theta^{\prime}$ as a function of angle $\theta$

For the triangle shown in Figure $4(\mathrm{~b}), K$ is the center of gear k1, $K^{\prime}$ is the center of gear k2, and $P$ is the pin attached to gear k1. Thus, sides $K K^{\prime}$ and $K P$ of the triangle are of constant length ( $a$ and $r$ respectively), while side $K^{\prime} P$ is variable, depending on the position of the pin inside the slot. The sine law states that:

$$
\frac{r}{\sin \left(180-\theta^{\prime}\right)}=\frac{a}{\sin \phi} \Rightarrow \sin \phi=\frac{a}{r} \sin \left(180-\theta^{\prime}\right)=\frac{a}{r} \sin (180-\theta-\phi) .
$$

Using the sum of angles formula

$$
\sin \phi\left(1+\frac{a}{r} \cos (180-\theta)\right)=\frac{a}{r} \sin (180-\theta) \cos \phi
$$

and setting

$$
x=1+\frac{a}{r} \cos (180-\theta), \text { and } y=\frac{a}{r} \sin (180-\theta)
$$

we get

$$
\sin ^{2} \phi x^{2}=y^{2}\left(1-\sin ^{2} \phi\right)
$$

from which we finally get

$$
\sin \phi=\frac{y}{\sqrt{x^{2}+y^{2}}}, \text { or } \phi=\arcsin \left(\frac{y}{\sqrt{x^{2}+y^{2}}}\right) .
$$

Since $\theta^{\prime}=\theta+\phi$ the relation of $\theta^{\prime}$ and $\theta$ is

$$
\theta^{\prime}=\theta+\arcsin \left(\frac{y}{\sqrt{x^{2}+y^{2}}}\right) .
$$

Thus, in simulating the rotation of the various gears, the position of gear k2, as defined by angle $\theta^{\prime}$ can be computed by the position of gear k1 as defined by angle $\theta$.

Another use of simulation modeling for verification of the mechanism's operation is the computation of the gear parameters. Both the old radiographs and the modern tomographies showed that the teeth of the Antikythera mechanism's gears were triangular. This teeth geometry is the easiest to manufacture, especially with the tools available at the time, but the least appropriate for smooth interlocking. The reason is clearly shown in Figure [5. If the distance of the gear centers 


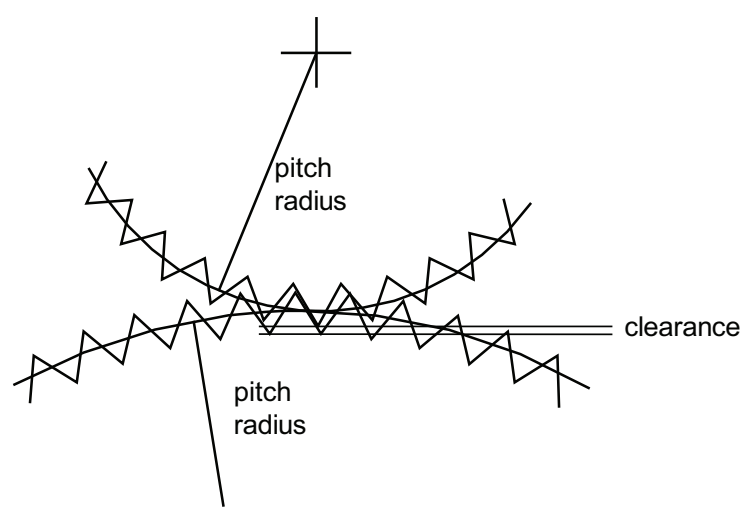

Figure 5: Gear parameters.

is such that the tip of a tooth falls very close to the land between the teeth, then the gears cannot rotate because the tip hits the side of the opposite tooth.

Depending on the actual inner (at the lands) and outer (at the teeth) diameters of the gears, what needs to be computed is the pitch radius of each gear. The pitch radius is defined as the radius at which two cylinders would roll on each other, assuming that the gears are replaced by touching cylinders. Taking into account that the cylinders cannot slide at all, the pitch ratio is exactly equal to the gear ratio. Thus, by specifying one of the pitch radii, the other one can be directly derived. If the clearance, defined as the distance between one tooth's tip from the opposite land, is too small, rotation is not possible as explained before. If the clearance is too large, then there is inadequate transfer of torque between the gears, resulting in a difficult or impossible operation of a long gear train. This is probably the reason that most of the physical models that are accurate reconstructions of the Antikythera Mechanism, do not operate smoothly if at all. On the other hand, computer simulation models can operate smoothly due to the precise calculation of the clearance between each pair of interlocking gears.

Since the main purpose of developing the simulations and animations of the Antikythera Mechanism was the validation and verification of the measurements obtained by either de Solla Price or the newer research, we tried to avoid the inclusion of any missing parts of the mechanism. This was not always possible, because the operation of some parts cannot be demonstrated without showing their interconnection to other parts. However, the insertion of missing parts for the interconnection was kept to the simplest form possible for two reasons: a) the mechanism is very elaborate and elegant, which implies that its craftsman would have gone to the simplest solution possible for any design problem he faced, b) a complicated solution may inadvertently include some engineering knowledge that was not available at the time of the mechanism's development.

This insistence for simplification of the missing parts forced us to include inaccuracies to the simulation rendering, instead of including complicated gear trains. This is illustrated by the rendering of the moon pointer's mounting on its axis, as shown in Figure 6. Only the crown shown in the figure was identified in the existing fragments of the mechanism. The small gear on the sun pointer's axis was not found. The problem is that the crown is, in reality, facing the other way around. This would require the inclusion of at least 3 more gears and a subframe to support them, in order to convert the horizontal rotation of the two pointers into the relative vertical rotation of 


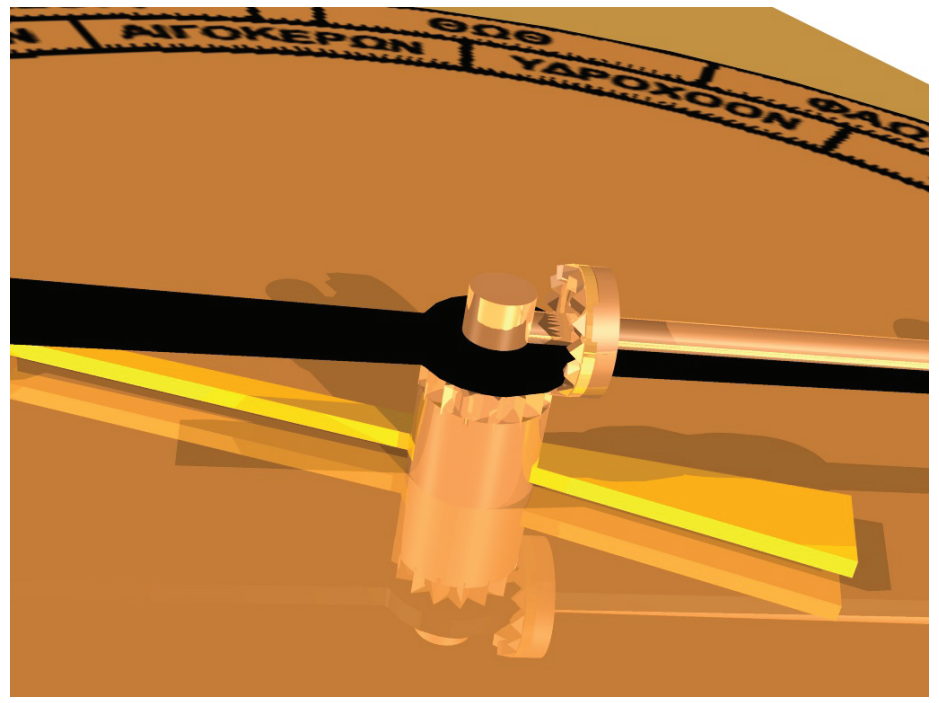

Figure 6: Rendering of the moon pointer mounting.

the moon's sphere that depicts the moon's phases.

The solution included in the simulation models is extremely simple and elegant, following the philosophy of the mechanism, even though is does not exactly correspond to the available fragments of the mechanism.

\section{Accessibility and Dissemination}

Apart from providing a tool for design validation, the computer simulation of the Antikythera Mechanism plays another equally important role: making the operation of the mechanism accessible to anyone, and disseminating the results of the research.

As mentioned in a previous paragraph, the first animation of the mechanism was developed around 1982 by Robert Morris. This simulation was not readily available, both because computer usage was not widespread at the time, and because it was developed on a limited availability block diagram compiler (ANIM8) [B]]. We created the first animations of the mechanism around 1998. They were based on de Solla Price's findings and were readily available over the Web. To get an indication of whether these animations increased awareness of the mechanism, we counted the number of researchers working on the mechanism. Data were taken from related publications in journals and conferences, as well as web references on the development of models and articles on the mechanism. The data are plotted in Figure $\square$.

Starting from 1950 when mainly de Solla Price was working on the mechanism [䧃], the number has increased very slowly with the addition of Wright, Bromley [W] and very few others. The first animation of the mechanism did not have an impact on the number of researchers mainly due to its virtually nonexistent availability. Our animations appeared on the web in 1998 [Q] from where they were freely downloadable. The diagram shows that this is a knee point, as the number of researches working on the Antikythera Mechanism started to increase exponentially afterwards, whereas before that, the increase was linear. 


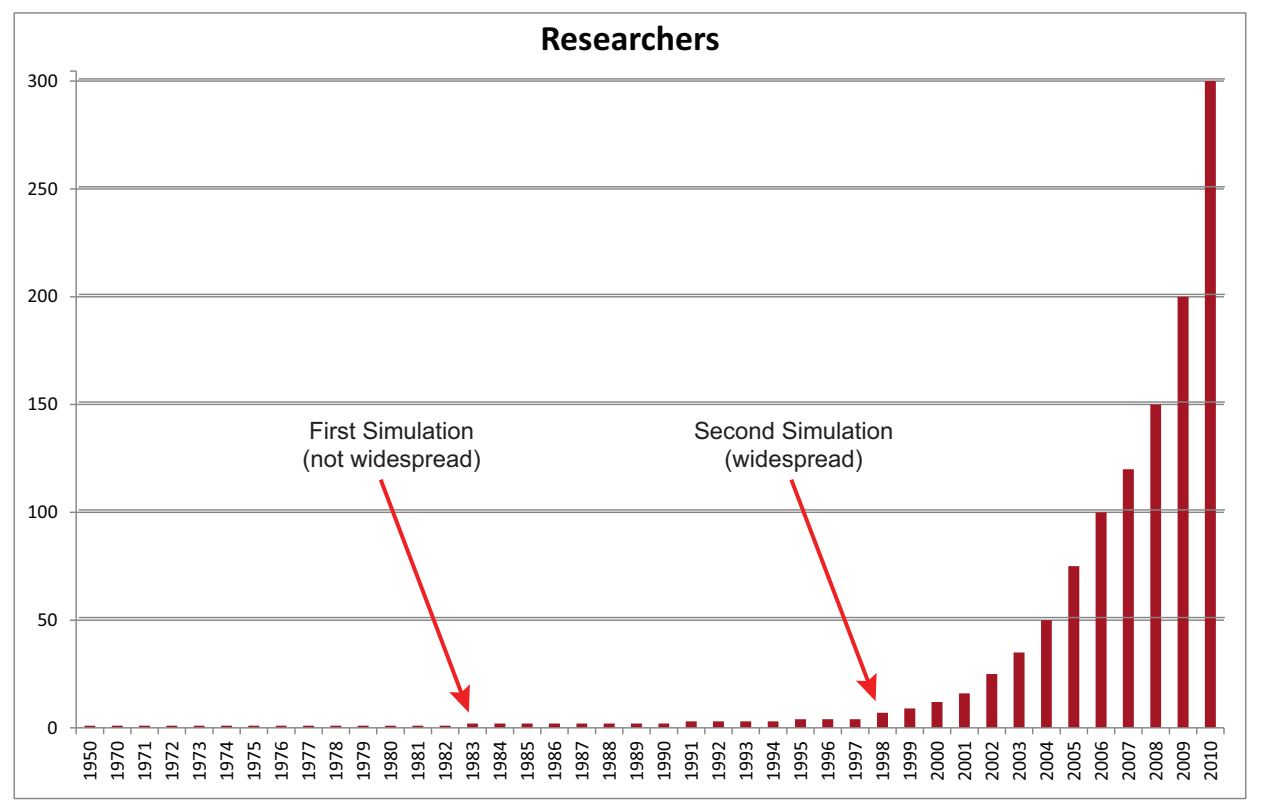

Figure 7: Number of researchers working on the mechanism vs. year.
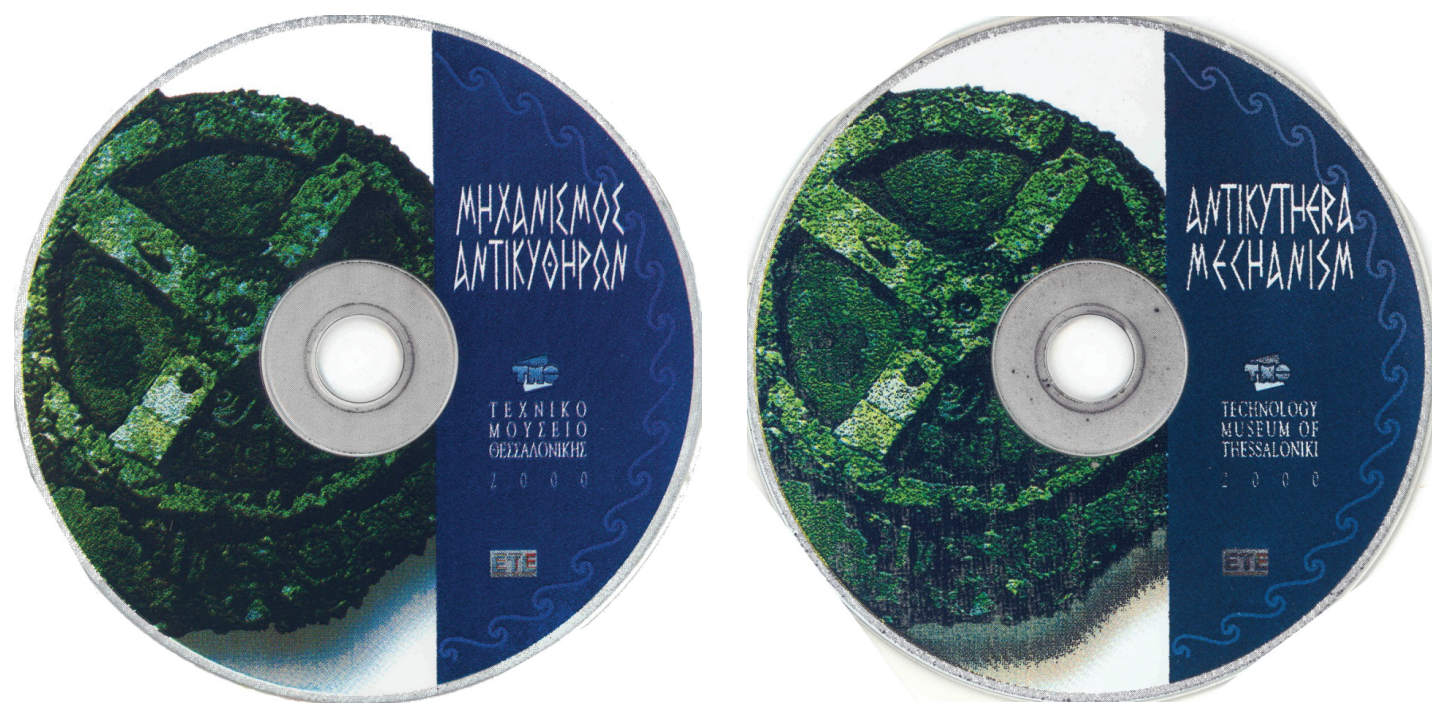

Figure 8: First edition of computer simulation, produced in 1999.

Dissemination of the animations through the web was augmented by the multimedia CDs produced by the Technology Museum of Thessaloniki (now called Noesis). These CDs (shown in Figure (8) included the animations along with the history and description of the mechanism, and were published in three languages: Greek, English, and German [ [8]. Following the late Prof. Economou's suggestion, the CDs were prepared in time for the EXPO 2000 in Hannover, Germany, and were given away at the Greek Pavilion.

The simulations of the Antikythera Mechanism were posted on the same site [Q] in 2005, again being freely downloadable. A frame of the simulation is shown in Figure $\mathbf{9}$. Response to the Web 


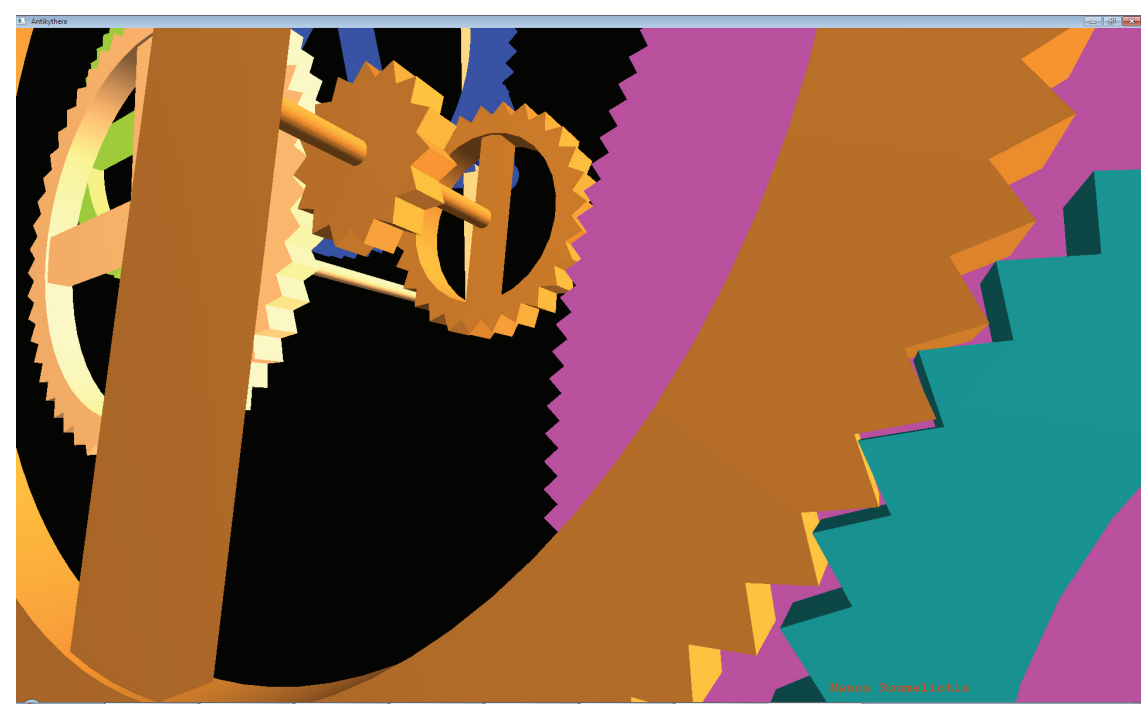

Figure 9: Simulation of the mechanism. "Zooming-in" to check clearance of the gears.

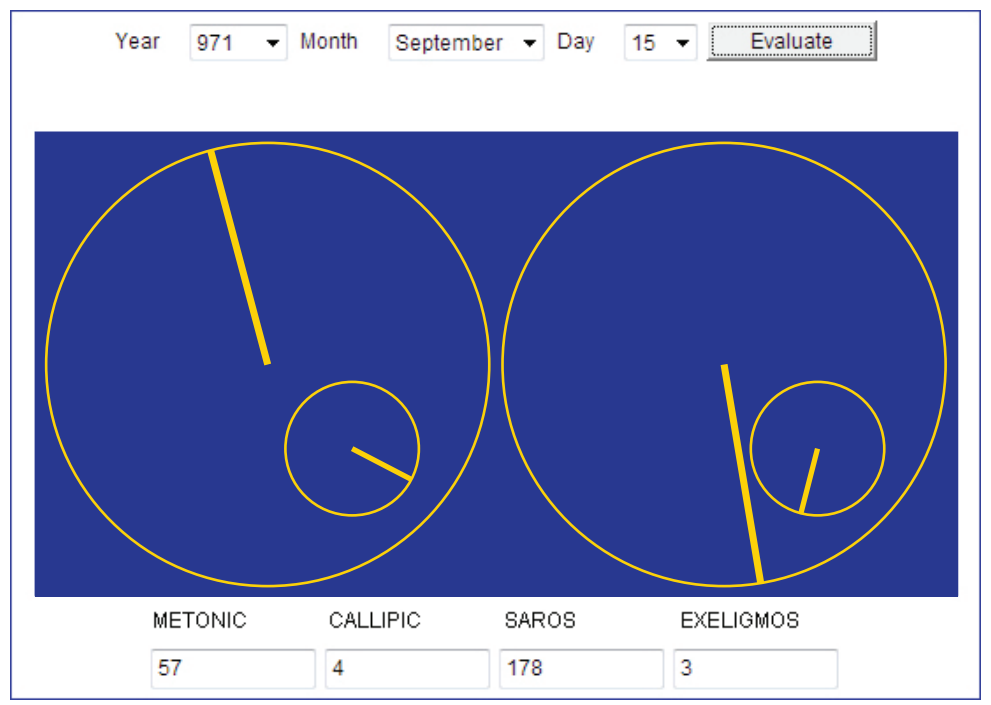

Figure 10: Simulation to compute the various astronomical cycles.

distribution of the animations and, subsequently, the simulations was overwhelming. The author received hundreds of emails asking for further information and requesting permission to use the animations and simulations for various publications. For example a composite picture of the animation appeared on the cover of engine [ए]] a magazine for teaching English to German engineers! Several of the people who contacted the author, subsequently became very active researchers on the mechanism themselves.

Apart from simulating the operation of the mechanism by developing a precise model, a more abstract simulation of the mechanism can verify the long term accuracy of the mechanism's calculations. Such a simulation is shown in Figure 10 . The user enters the date and the simulator computes the position of the four cycles calculated by the Antikythera mechanism, namely the 
Metonic, Callipic, Saros, and Exeligmos cycles. The computation is not performed by solving equations, but by "turning" the gears of the mechanism as many times as required to reach the date set by the user, and then "reading" the back dials of the mechanism.

\section{Conclusions}

This paper discussed the computer simulation models of the Antikythera Mechanism and compared them to the physical models of the device. It was shown that simulations have certain advantages over the physical models, namely the verification of estimated parameters and validation of hypotheses about the mechanism's design. In addition, computer simulation models helped in achieving widespread awareness of the mechanism, greatly enhancing appreciation of Ancient Greek technology.

\section{References}

[1] K. Efstathiou, A. Basiakoulis, M. Efstathiou, M. Anastasiou, J.H. Seiradakis, "Determination of the gears geometrical parameters necessary for the construction of an operational model of the Antikythera Mechanism," Mechanism and Machine Theory, Volume 52, June 2012, Pages 219-231.

[2] T. Freeth, Y. Bitsakis, X. Moussas, J. Seiradakis, A. Tselikas, H. Mangou, M. Zafeiropoulou, R. Hadland, D. Bate, A. Ramsey, M. Allen, A. Crawley, P. Hockley, T. Malzbender, D. Gelb, W. Ambrisco, and M. Edmunds, "Decoding the ancient Greek astronomical calculator known as the Antikythera Mechanism," Nature, Vol. 444, No 11, November 2006, pp. 587-591.

[3] R.I. Morris, "Derek de Solla Price and the Antikythera Mechanism: an Appreciation," IEEE Micro, Vol. 4, No 1, February 1984, pp. 15-21.

[4] D. de Solla Price, "An ancient Greek computer," Scientific American, Vol. 201, June 1959, pp.60-67.

[5] D. de Solla Price, "Gears from the Greeks: The Antikythera Mechanism - A Calendar Computer from ca. 80 B," Transactions of the American Philoshophical Society, Vol. 64, Part 7 (new series), Nov. 1974.

[6] D. de Solla Price, "The Productivity of Research Scientists," 1975 Yearbook of Science and the Future, Encyclopaedia Britannica, Inc., Chicago, 1975, pp. 409-421.

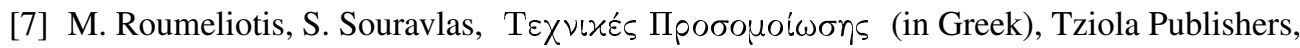
Thessaloniki, Greece, 2012.

[8] M. Roumeliotis, Antikythera mechanism, Multimedia CD (in Greek, English and German), Technology Museum of Thessaloniki, Thessaloniki, Greece, 2000.

[9] M. Roumeliotis, "Simulation and Animation of the Antikythera Mechanism," Web 2012 $<\mathrm{http} / / /$ www.etl.uom.gr/mr/index.php?mypage=antikythera $>$.

[10] W. Rybczynski, "A Greek Time Machine," Engine, March 2005, pp. 58-61.

[11] M.T. Wright, A.G. Bromley, "Current Work on the Antikythera Mechanism, " Proceedings of the

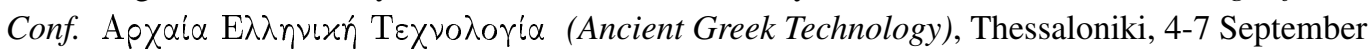
1997, pp. 19-25. 